\title{
Do intercultural factors play a role in exacerbating psychiatric symptoms?
}

Yong Lock $\underline{O n g}^{1}$, MBBS, FRCPsych, Hwa Ling $\underline{\text { Yap }}^{1}$, MBBS, MMed

ABSTRACT We report the case of a 29-year-old mixed-race woman suffering from recurrent major depressive episodes, with suicidal ideation and risk, involving several inpatient admissions. A comorbid diagnosis of borderline personality disorder was also recorded in one of her previous inpatient admissions. During her last inpatient admission, a multidisciplinary case discussion and review of the patient's life highlighted several possible intercultural trigger factors that could have contributed to the exacerbation of her psychiatric illness. We emphasise the need to explore intercultural predisposing and precipitating factors for a more complete psychodynamic understanding of psychiatric illnesses among the multiracial population of Singapore. This also adds to the discussion on the management of such patients with the option of formal in-depth psychotherapy in adjunct to medication. This may prevent recurrent relapses, modify suicide intent and reduce the necessity for inpatient treatment, which will be cost-effective and result in efficacious treatment.

Keywords: culture, depression, personality disorder, treatment options

Singapore Med J 2013; 54(1): e16-e17

\section{INTRODUCTION}

Most Singaporeans correctly pride themselves on having a high tolerance and acceptance of racial and intercultural differences, and strive towards the Arcadian goal of peaceful multiculturalism. (1) There is, however, a risk of ethnic backgrounds taking precedence over their Singaporean identity, potentially leading to a backslide into a segregated society. This risk was identified by an opposition Member of Parliament. ${ }^{(2)}$ There is the impression that traditional ethnic values and behavioural norms held consciously, subconsciously or unconsciously by the diverse population is mitigated, to a large extent, by a higher incidence of interracial marriage among the current Singaporean population. However, these same values and behavioural norms may act as triggers for psychiatric symptoms and illnesses. This case report sets out to highlight the latter.

\section{CASE REPORT}

A 29-year-old mixed-race woman (biological father: Caucasian; biological mother: Malay), born an illegitimate baby, was adopted as the youngest and sixth child by a Malay family. Two of her five older siblings were also adopted. Her adoptive father died when she was three years old. In 2004, in the first of her multiple psychiatric inpatient admissions, she was diagnosed with major depressive illness with suicidal ideation. Subsequent admissions recorded attempts to contain and treat her depression and repeated drug overdoses. She was treated with a combination of antidepressants, antipsychotics, benzodiazepines and electroconvulsive therapy (ECT). A comorbid diagnosis of borderline personality disorder was also recorded in one of her admissions.
There was documented evidence of early abuse during childhood, and an inpatient episode in 2008 was thought to be the result of an alleged abuse by the patient's brother-in-law. Entries made in the medical notes during that admission recorded her brother-in-law entering the patient's bedroom (shared with her adoptive mother) and described him standing by her bed, staring intensely at her sleeping form. As a result, she moved out of her family home and was accused of being disloyal to the family unit. She had by then achieved the professional status of a teacher. In 2008, there was also documented evidence of the patient's biological mother being admitted to the Institute of Mental Health for bipolar affective disorder. The patient subsequently began a relationship with a Chinese man. As he was unable to convert to Islam due to his job specifications, the couple was unable to marry in a traditional bersanding (enthronement) ceremony and was instead married in a civil marriage ceremony. Shortly, the patient had a relapse of a major depressive episode, warranting her latest inpatient admission in August 2011.

It is believed that the institution of adoption has been firmly established as an integral part of the Malay family structure in Singapore. Although the transfer of children from their birth homes to their adoptive homes is usually conducted with minimal distress to the parents and children involved, ${ }^{(3)}$ the present case carries the additional stress variables of illegitimacy and mixed-race parentage. Despite improved education and increased professional status among women in Singapore, as seen in the patient's training as a teacher, she continued to stay with her adoptive family, a behavioural pattern strongly recognised as the value orientations inculcated in Malay children to maintain the extended nuclear family structure. ${ }^{(4)}$ Her decision to leave

${ }^{1}$ Department of Psychological Medicine, Changi General Hospital, Singapore

Correspondence: Dr Ong Yong Lock, Principal Staff Attending Physician, Department of Psychological Medicine, Changi General Hospital, 2 Simei Street 3, Singapore 529889. ongyonglock@hotmail.com 
home due to a new incident of abuse was thus seen as an act that went against traditional values. Moreover, the patient had a long-term relationship with a Chinese man who could not convert to Islam, which subsequently resulted in the couple legalising their marriage in a civil marriage ceremony instead of a bersanding ceremony. Considered by most Malays to be a symbol of the 'true sanctity' of a Muslim marriage, the bersanding ceremony involves family and friends in an elaborate ceremony filled with symbolic traditions. Thus deprived of this cultural tradition, the patient relapsed into major depressive illness, requiring inpatient admission.

\section{DISCUSSION}

One of the strengths of psychiatry, unlike other medical and surgical specialties, is its ability to retain a certain degree of ambiguity with regard to the predisposing and possible contributing aetiological factors of an individual's illness. This allows time for reflection of the causes of a patient's inner turmoil as well as his or her cognitive schema. However, there is no speculative element when establishing the criteria for diagnosis, as defined by Diagnostic and Statistical Manual of Mental Disorders, 4th edition (DSM-IV) and International Classification of Diseases 10th revision (ICD-10).

Our patient suffered from recurrent major depressive episodes and was at high suicide risk during some of these episodes. The concrete predisposing factors for her illness include genetic loading from her biological mother for an affective disorder, the loss of her adoptive father at three years of age and the complete absence of a biological father in her life. There are many easily accessible evidence-based references regarding genetic disposition in depression, thus making any reference unnecessary here. One study that focused on the impact of the loss of fathers on girls during childhood has revealed that in womanhood, these girls exhibited greater insecurity and more negative self-evaluation, as compared to women who had not lost their fathers in childhood. ${ }^{(5)}$

With specific consideration of the cultural factors involved in this case report, adoption into Malay families is common and usually not problematic. However, it is tempting to hypothesise that illegitimacy, mixed-race parentage and the fact that one is markedly different may loosen the cultural norms surrounding the protective upbringing of such children. This leads to the difficult question of abuse in Malay families. There is a strong impression among social workers and the legal profession that Malay families are more frequently involved in behavioural patterns of abuse within the family, as compared to other racial groups. Ironically, there are no easily accessible evidence-based studies to substantiate this. However, this is still a sensitive and moot point. Our patient claimed to have been abused by a person in her extended family as a child and again as a young woman.

It is well documented that early abuse underpins a great deal of psychopathology. In particular, Waseem et al's study, which shows the correlation between child abuse and dissociative identity disorder, ${ }^{(6)}$ highlighted the destructive impact of child abuse and facilitated further discussion. Our patient, who had been diagnosed to be suffering from comorbid borderline personality disorder in one of her multiple inpatient admissions, could have just as easily developed a different personality disorder. Moreover, it would be unusual if our patient's personality had not been affected by her early experiences, however strong her underlying ego strengths may be. The different personality disorders that may develop from child abuse and neglect, as discussed in the current literature, can be considered a useful point for the continuing medical education of psychiatrists.

The main diagnosis in this present case was recurrent depressive illnesses associated with high risk of suicide. The patient's latest episode of depression could have been triggered by another cultural factor - her marriage to a Chinese non-Muslim and her civil marriage ceremony. Could this deprivation of a traditional ceremony, considered to be of great importance among the Malay community, have played a part in her relapse? Our multidisciplinary team opined that it was a possible trigger, which reopened a discussion on the use of treatment options such as formal short-term dynamic interpersonal psychotherapy and problem-solving or cognitive behavioural therapy as adjuncts to medication and ECT in similar cases. Our patient, who was successfully treated with ECT in her most recent inpatient admission, was discharged well and followed up as an outpatient for the management of depression, as well as the assessment of her suitability for one of the above adjunct therapies.

In conclusion, the dynamic interplay of disrupted traditional Malay values, combined with an input of Chinese culture and an unknown genetic shadow of the Caucasian biological father, may be the cultural factors that had exacerbated the patient's psychiatric symptoms. This also raises the importance of empathy toward and exploration of intercultural issues in multiracial Singapore, as well as the need for talking therapies in addition to medication, in order to promote better understanding and resolution of intercultural triggers and the resulting symptoms.

\section{REFERENCES}

1. Henschke R. Singapore: The Secret of Multi-racial Harmony. In: Asia Calling [online]. Available at: www.asiacalling.kbr68h.com/ur/ archives/1524-singapore-the-secret-of-multi-racial-harmony. Accessed November 2, 2011.

2. Giam G. Singapore: Multiculturalism or the Melting Pot? [online]. Available at: geraldgiam.sg/2009/07/singapore-multiculturalism-ormelting-pot/. Accessed November 2, 2011.

3. Djamour J. Malay Kinship and Marriage in Singapore. London: Atholone Press, 1959.

4. Tham SC. Malay Family Structure: Change and Continuity with Reference to Singapore. Singapore: National University of Singapore, 1993.

5. Young ER, Parish TS. Impact of father absence during childhood on the psychological adjustment of college females. Sex Roles 1977; 3:217-27.

6. Waseem M, Aslam M, Switzer RM Jr, Perales O. Child abuse and neglect: Dissociative Identity Disorder. In: Medscape Reference [online]. Available at: emedicine.medscape.com/article/916186-overview. Accessed November 2, 2011. 